\title{
In vivo observations and in vitro experiments on the oral phase of swallowing of Newtonian and shear-thinning liquids
}

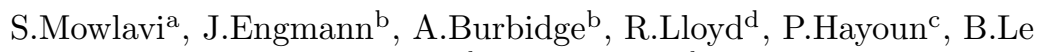 \\ Reverend $^{\mathrm{b}}$, M.Ramaioli ${ }^{\mathrm{d}, *}$ \\ ${ }^{a}$ Department of Mechanical Engineering, Massachusetts Institute of Technology, 77 \\ Massachusetts Av., Cambridge MA 02139, USA \\ ${ }^{b}$ Nestlé Research Center, Route du Jorat 57, 1000 Lausanne 26, Switzerland \\ ${ }^{c}$ CNRS/UPMC/ESPCI ParisTech-PSL Research University, UMR 7615, Laboratoire \\ SIMM, 10 Rue Vauquelin, 75231 Paris, France \\ ${ }^{d}$ Department of Chemical and Process Engineering, Faculty of Engineering and Physical \\ Sciences, University of Surrey, Guildford, GU2 7XH, United Kingdom
}

\begin{abstract}
In this study, an in vitro device that mimics the oral phase of swallowing is calibrated using in vivo measurements. The oral flow behavior of different Newtonian and non-Newtonian solutions is then investigated in vitro, revealing that shear-thinning thickeners used in the treatment of dysphagia behave very similarly to low-viscosity Newtonian liquids during active swallowing, but provide better control of the bolus before the swallow is initiated. A theoretical model is used to interpret the experimental results and enables the identification of two dynamical regimes for the flow of the bolus: first, an inertial regime of constant acceleration dependent on the applied force and system inertia, possibly followed by a viscous regime in which the viscosity governs the constant velocity of the bolus. This mechanistic understanding provides a plausible explanation for similarities and differences in swallowing performance of shear-thinning and Newtonian liquids. Finally, the physiological implications of the model and experimental results are discussed. In vitro and theoretical results suggest that
\end{abstract}

\footnotetext{
* Corresponding author

Email address: m.ramaioli@surrey.ac.uk (M.Ramaioli)
} 
individuals with poor tongue strength are more sensitive to overly thickened boluses. The model also suggests that while the effects of system inertia are significant, the density of the bolus itself plays a negligible role in its dynamics. This is confirmed by experiments on a high density contrast agent used for videofluoroscopy, revealing that rheologically matched contrast agents and thickener solutions flow very similarly. In vitro experiments and theoretical insights can help designing novel thickener formulations before clinical evaluations.

Keywords: Bolus, Flow, Swallowing, Tongue, Ultrasound, Viscosity, Thickener, Rheology, Fluid mechanics, Oral cavity, Palate, Peristalsis.

2010 MSC: 00-01, 99-00

\section{Introduction}

Swallowing disorders (dysphagia) can be caused by several conditions such as stroke, dementia, neurological diseases or head and neck cancer. Dysphagia is considered the main cause of post-stroke pneumonia that affects about $20 \%$ 5 of stroke patients (Scheitz et al. 2015) and causes more than $10 \%$ of post-stroke deaths (Koennecke et al., 2011). More generally, in elderly dysphagia can result in food and drink avoidance leading to malnutrition and dehydration.

Several approaches exist to manage dysphagia, one of the most popular being the use of thickeners to increase the viscosity of thin drinks, resulting in shear-thinning liquids. A review of their efficacy and limitations can be found in Newman et al. (2016). Despite the clinically proven efficacy of thickeners, it remains unclear, at least from a mechanistic perspective, exactly why they are effective and what role is played by their shear-thinning rheology. Consequently it is difficult to either develop better thickeners, or to identify which patients are likely to respond to which level of viscosity other than by trial and error and there is a strong ongoing debate within the medical community about what represents best practice. Some would argue that prescribing overly thickened beverages can lead to excessive residues in the pharynx and oral cavity, which, 
although providing a safe initial swallow, are likely to provide post-swallow complications.

Clearly, the physical act of forming and then swallowing a liquid bolus is exquisitely complicated and requires precise coordination of many muscles in order to avoid catastrophic failure. From the mechanistic standpoint, the kinematics and dynamics of single muscles involved in mastication and/or swallow25 ing are still the object of active research (Weickenmeier et al., 2016) and it is a formidable challenge to describe realistically all organs and tissues moving during these food oral processes.

The clinical observation of subjects is usually carried out by one of two popular techniques: fiberoptic endoscopic evaluation of swallowing (FEES, see

30 Hiss and Postma, 2003), which involves insertion of an instrumented catheter through the nose, or videofluoroscopic swallowing study (VFFS, see Logemann, 1998), which is an X-ray based video technique. Both of these techniques are cumbersome and invasive: insertion of a catheter will certainly perturb the flow, and likely the physiology, and radiation exposure limits repeats and studies on healthy individuals. There are several other techniques available, but they also have significant drawbacks. For this reason, the concept of in vitro swallowing experiments is beguiling, and many things can be learned from this approach, despite the difficulty of matching the biophysical reality exactly. In this article an in vitro 'swallowing simulator' is presented, which mimics some aspects of

40 the in vivo swallow, and enables quantitative measurements and comparisons of the oral flow of different types of liquids. To be absolutely clear, this should not be considered as an alternative to clinical research. However, judicious use of in vitro experiments like those detailed here could allow for improved understanding of the relative significance of different effects, leading to stronger 45 and tighter focused hypotheses that can subsequently be tested in vivo.

To put our current findings into context, we first present a very short review of the most relevant literature. 
A simple and mechanistic theory of the oral phase of swallowing was initially proposed by Nicosia and Robbins (2001). This theory considered the transient motion of two approaching parallel plates as a model for the tongue and the palate. De Loubens et al. $(2010,2011)$ later introduced an in vitro pharyngeal peristalsis simulator and a theory to study the thin film left on the pharyngeal mucosa after a swallow, this time using two rolling cylinders to model the peristaltic action of the tongue against the pharyngeal wall.

The above studies only considered the flow of Newtonian liquids and did not address the behavior of thickened solutions. Popa Nita et al. (2013) used rotational rheometry to characterise the rheology of aqueous solutions of Resource ${ }^{\circledR}$ ThickenUp ${ }^{\mathrm{TM}}$ Clear (TUC), a common dysphagia thickener, and demonstrated that their steady state viscosity is close to that of Bracco $^{\mathrm{TM}} \mathrm{E}-\mathrm{Z}-\mathrm{PAQUE}$, a common video fluoroscopy contrast agent. However, their study did not discuss the relevance of using steady state rheological properties for a transient, fast flow such as swallowing.

Mackley et al. (2013) used different techniques to characterise the rheology of several dysphagia thickeners and introduced for the first time a qualitative, 'in vitro swallowing simulator' mimicking the oral phase of swallowing. Building upon this first device, Hayoun et al. (2015) proposed a more quantitative in vitro experiment, devised a theoretical model and applied both to studying the flow of Newtonian liquids.

This study extends the scope of the latter by using in vivo observations to determine an appropriate value of the mechanical inertia of the in vitro swallowing simulator. The behavior of shear-thinning liquids is then investigated in the calibrated device and compared to that of Newtonian liquids. Finally, the theoretical model is extended and refined and a novel mechanistic interpretation of the experimental results is proposed through the identification of two 75 dynamical regimes. This interpretation also leads to a novel explanation for the role played by the shear-thinning rheology of thickened fluids in managing 
dysphagia.

This study is presented as follows. In section 2 we describe the experimental device, select test fluids and the in vivo experiments used to calibrate the swallowing simulator. Section 3 describes the full theory and a simplified model. Section 4 describes the results obtained, the implications of which are then discussed is section 5 before drawing some conclusions in section 6 .

\section{Materials and methods}

\subsection{In vitro swallowing simulator}

During this study we further developed a mechanical, in vitro device designed to study and understand the flow of a liquid induced by the movement of the tongue during the oral phase of swallowing. This device, shown in Figure 1 is based on a mouth-sized in vitro experiment initially designed by Mackley et al. (2013) and later improved by Hayoun et al. (2015). Without having

90 the ambition of reproducing faithfully the in vivo process, the objective of this study was to capture two essential features of the mouth and tongue. A thin and flexible polyethylene membrane is attached to the "palate" and contains the liquid, representing the oral cavity. A roller attached to a rotating arm is driven by a weight and mimics the driving pressure applied by the tongue to the bolus, propelling the liquid through the oral cavity. The effect of gravity on the rotating arm is cancelled out by a counterweight. Different driving forces $F$ lead to different pressures applied on the liquid, which can be computed as illustrated in Hayoun et al. (2015).

A significant improvement made to the model experiment in this current study, with respect to the experiment used in Hayoun et al. (2015), consists of the possibility of varying the total moment of inertia $I_{t o t}$ of the rotating arm, roller and counterweight assembly. To that effect, two radially opposed rods, each carrying an offset mass, can be attached to the rotating arm. In the 


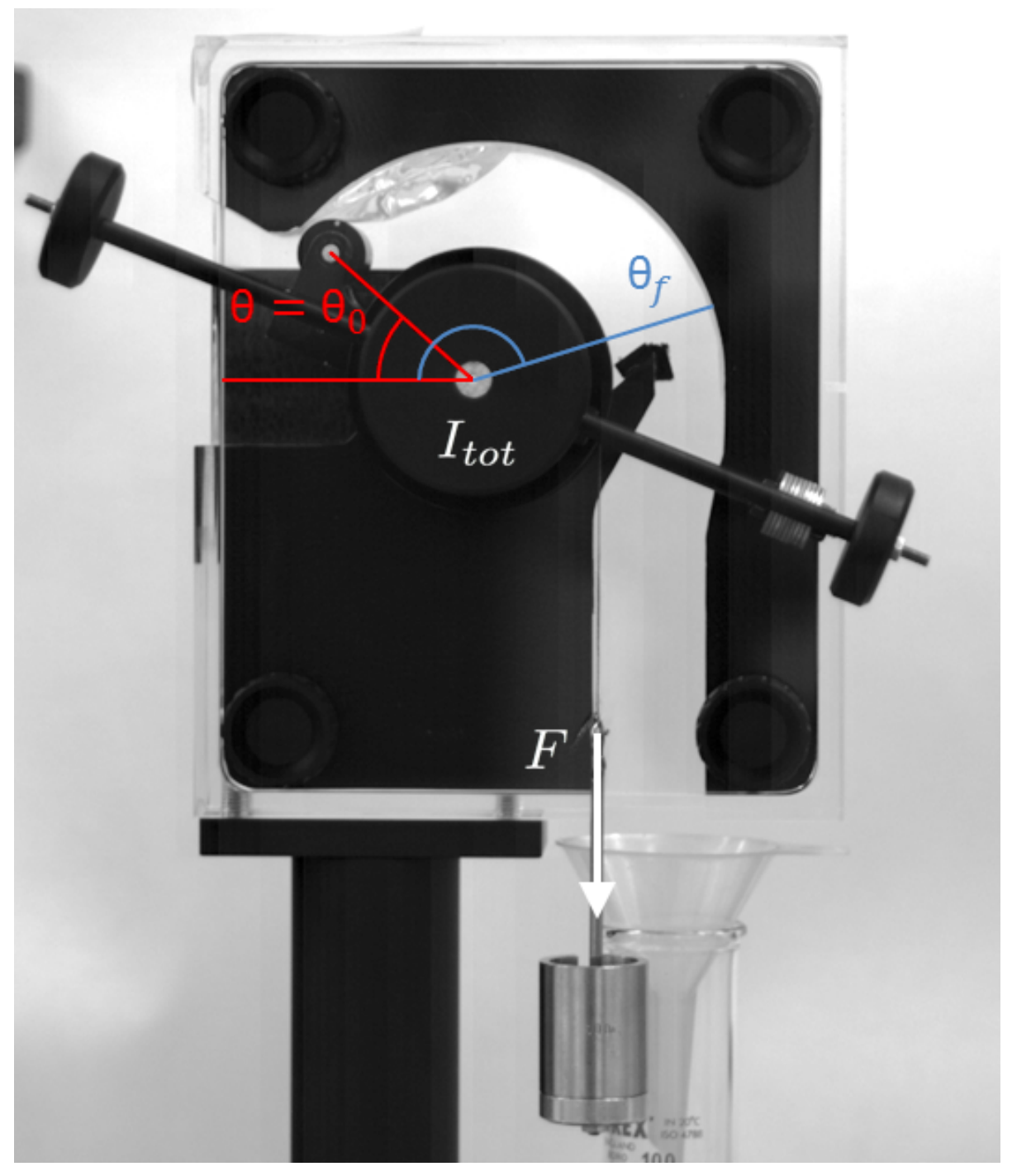

Figure 1: The in vitro experiment showing the roller at the initial position $\theta=\theta_{0}$ and the two radially opposed offset masses to vary the moment of inertia $I_{t o t}$ of the rotating arm, roller and counterweight (in this image $I_{\text {tot }}=1.15 \cdot 10^{-3} \mathrm{~kg} \mathrm{~m}^{2}$ ). The weight imposes a driving force $F$ represented by the white arrow. 
physiological context, $I_{t o t}$ accounts for the combined inertia of all the tissues and organs involved in a swallow, including the tongue, the larynx, the hyoid bone and the associated muscles and soft tissues. Due to the non-rigid body motion, the inertia of the tissues is difficult to estimate and we take the approach of calibrating $I_{\text {tot }}$ using in vivo observations, as described later in Section 4 . Other improvements to the in vitro device include the use of an inert polyethylene film instead of a porous dialysis tube, and the use of an inextensible steel string to hold the weight.

The time evolution of the position of the liquid during an in vitro experiment was quantified by measuring the angle $\theta$ between the roller and the horizontal direction, as shown in Figure 1. To carry out an artificial swallow experiment, 115 a closed flexible membrane was first attached to the "palate" along the path of the roller. $6 \mathrm{ml}$ of liquid were then injected into the front opening of the membrane and the liquid was manually pushed through the membrane until it reached its starting position, shown in Figure 1. A pin initially held the roller in its starting angular position $\theta_{0}=45^{\circ}$. Releasing the pin caused the roller, driven by the weight, to propel the bolus inside the flexible membrane until the liquid was ejected at the open end, and the roller reached its final position $\theta_{f}=165^{\circ}$. The roller motion was recorded at a frame rate of $500 \mathrm{fps}$ with a high speed camera (Photron FastCam SA3) and an automated image analysis extracted the time evolution of the roller angular position $\theta$ from the video recordings. The oral transit time $t_{\text {end }}$ was defined as the time taken by the roller to reach the angle $\theta_{\text {end }}=120^{\circ}$ and the exit velocity $v_{e}$ was defined as the linear velocity of the roller when it reaches $\theta_{\text {end }}$. We typically ran 3 to 4 swallows per solution and observed good reproducibility of the results. More details about the experimental method can be found in Hayoun et al. (2015). 


\subsection{In vivo measurements}

In vivo measurements were realized using a Siemens Acuson SC2000 echographer and a 8v3c curvilinear probe. Subjects were asked to swallow liquid boluses of volume $10 \mathrm{ml}$ in one go, whilst holding an ultrasonic probe against their throat. The device allowed either to record a video of the bolus flow during the swallow, or to measure the velocities of the fluid by Doppler effect. Video recordings were carried out on a single subject with three repetitions per liquid viscosity during the development of the method that conducted to the preclinical phase of the clinical trial with reference 03/12, approved by the Commission Cantonale d'Éthique de la Recherche Sur l'Être Humain, Vaud, Switzerland. Velocity measurements were undertaken on ten subjects with two repetitions per liquid viscosity, as part of the above mentioned clinical trial. For these in vivo experiments, the oral transit time $t_{\text {end }}$ was defined as the time taken by the bolus to completely disappear from the ultrasound field of view. This position corresponds to $\theta_{\text {end }}$, introduced above. The in vivo exit velocity $v_{e}$ is the average liquid velocity measured when the bolus is at $\theta_{\text {end }}$.

\subsection{Materials}

Aqueous glycerol solutions of various concentrations were used as model Newtonian fluids in the in vitro experiment. Their rheology was characterized for shear rates from $10^{-1}$ to $500 \mathrm{~s}^{-1}$ at $22^{\circ} \mathrm{C}$ with an Anton Paar MCR 500 rheometer equipped with a concentric cylinder geometry $(\mathrm{OD}=28.9 \mathrm{~mm}, \mathrm{ID}=$ $26.6 \mathrm{~mm})$. Varying the glycerol concentration from $50.0 \%$ wt to $99.8 \%$ wt results in a range of viscosities going from $\eta=6 \mathrm{mPa}$ s to $1185 \mathrm{mPa} \mathrm{s}$, as shown by the horizontal straight lines in Figure 2. Because glycerol is not edible, in vivo measurements with Newtonian liquids were performed using aqueous molasses,

55 the concentrations of which were adjusted in order to match the viscosities of the glycerol solutions. 
Aqueous solutions of Resource ${ }^{\circledR}$ ThickenUp ${ }^{T M}$ Clear (TUC), a dysphagia thickening powder were also investigated. TUC contains xanthan gum as the main thickener, as well as maltodextrin as an excipient. This gives rise to non-

160 2013). In this study, TUC solutions were prepared with deionized water in different concentrations that are commonly used by dysphagic subjects, namely $0.6 \%$ wt to $3.6 \%$ wt. These concentrations were used both in the in vitro experiment and in vivo measurements. Finally, a ready to use contrast agent

\section{Theoretical model}

\subsection{Full theoretical model}

Hayoun et al. (2015) developed a mechanical theory to interpret the results of the in vitro experiments, which was further developed as follows. The rotation of the arm assembly, the bolus and the suspended weight can be described by Newton's second law, leading to the following differential equation governing the time evolution of the roller angular velocity $\omega=\dot{\theta}$ :

$$
\begin{aligned}
\left(I_{\text {tot }}+I_{\text {bolus }}+\frac{F}{g} r_{C}^{2}\right) \dot{\omega}= & r_{C}\left(F-F_{\text {min }}\right)-r_{B} F_{d}(\rho, \eta, \omega) \\
& -r_{B} \rho V g \cos \left(\theta+\frac{L}{2 r_{B}}\right),
\end{aligned}
$$

where $I_{t o t}$ is the moment of inertia of the rotating arm, roller, counterweight and (possibly) offset masses assembly, $I_{b o l u s}$ that of the bolus with respect to the 


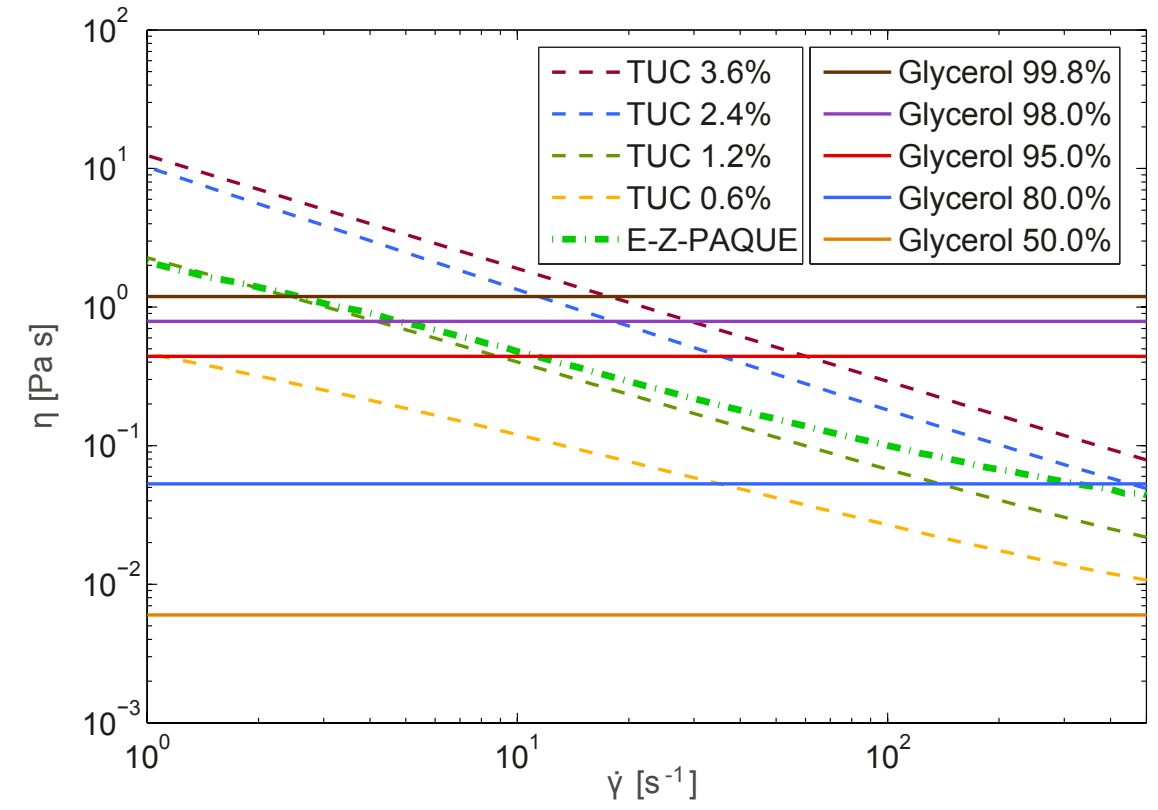

Figure 2: Shear viscosity versus shear rate for the various Newtonian aqueous solutions of glycerol, shear-thinning solutions of TUC and for E-Z-PAQUE $41 \% \mathrm{w} / \mathrm{w}$. Measurements were performed at $22^{\circ} \mathrm{C}$. 
the wire. The bolus density and viscosity are denoted by $\rho$ and $\eta$, respectively. $F_{d}$ is the force due to the viscous drag of the bolus. Other variables include the "throat" radius $r_{B}=0.051 \mathrm{~m}$ and the radius $r_{C}=0.028 \mathrm{~m}$ of the pulley wheel to which the mass is attached. $V=6 \cdot 10^{-6} \mathrm{~m}^{3}$ and $L=0.050 \mathrm{~m}$ represent respectively the volume and length of the bolus. Finally, $F_{\min } \sim 0.3 \mathrm{~N}$ is an experimentally observed minimum driving force, independent of the type of liquid.

The theory presented in Hayoun et al. (2015) was improved by considering the effect of gravity, represented by the last term in Equation (1). In order to take into account the large range of Reynolds numbers possible in the bolus flow, the dissipation force $F_{d}(\rho, \eta, \omega)$ is now computed from a computational fluid dynamics (CFD) simulation of the flow within the bolus, as described in the Appendix. This leads to the following expression for the dissipation force

$$
F_{d}(\rho, \eta, \omega)=\alpha \rho r_{B}^{2} \omega^{2} H^{2} f(R e)
$$

where $R e=\rho H r_{B} \omega / \eta$ is the Reynolds number of the flow within the bolus, $f(R e)$ is the dimensionless dissipation force retrieved from the CFD and $\alpha=$ 10.6 is a correction factor that was obtained by fitting the CFD results to the experimental data.

The theory shows that the dynamics of the bolus involves not only the flow within the bolus through the dissipation force, but also the motion of the rotating arm. It is thus important to consider the relative sizes of the bolus fluid inertia $I_{\text {bolus }}$ and the inertia $I_{\text {tot }}$ of the device itself, which we will refer to as system inertia. The fluid inertia is important in determining the apparent Reynolds number of the bolus flow, but it is very small relative to the inertia of the whole system. In the real, physiological context, the system inertia is a proxy for the mass of the tongue, hyoid bone and pharynx, which clearly is highly significant in controlling the overall dynamics, although very difficult to directly measure. Later, we will obtain an approximate value for this by 
calibrating against in vivo flow measurements.

This analysis also shows that the Reynolds number is not sufficient to describe the dynamics of the bolus, since it only considers the flow within the bolus, hence affecting only the dissipation force. In the next section, a simplified theory enables the identification of different dynamical regimes for the bolus and we introduce a relevant dimensionless variable for the description of these regimes.

\subsection{Simplified theoretical model}

In the following section, it will be shown that the full theory introduced in Equation (1) shows very good quantitative agreement with the experimental results. However, the presence of the numerically computed dissipation force $F_{d}(\rho, \eta, \omega)$ does not allow for an analytical solution of the equation. We therefore introduce in this section a simplified, qualitative version of this model that can be solved in closed form, which provides us with considerable insight into the dynamics of the system. The qualitative relevance of this simplified model will be validated in Section 5 by comparing its predictions with results from the full theory and experiments.

The simplified theory relies on the three following simplifications: (i) we consider the low- $R e$, linear expression for the dissipation force $F_{d}$, (ii) we neglect the minimum driving force $F_{\min }$ and (iii) we neglect the gravitational force on the bolus. These simplifications, which do not alter the qualitative relevance of the model in describing the dynamics of the bolus, lead to the following differential equation :

$$
\left(I_{\text {tot }}+I_{\text {bolus }}+\frac{F}{g} r_{C}^{2}\right) \dot{\omega}=r_{C} F-\alpha q_{1} H r_{B}^{2} \eta \omega,
$$

where $q 1=74.18$ results from a fitting of the CFD results described in the 220 Appendix at low $R e$. 
The following analytical solution for the time-dependent dynamics of the bolus can be conveniently obtained :

$$
\omega(t)=\omega^{*}\left(1-e^{-t / t^{*}}\right),
$$

where $t$ is the time since the beginning of the swallow, and the characteristic time $t^{*}$ and velocity $\omega^{*}$ are defined as

$$
t^{*}=\frac{I_{t o t}+I_{\text {bolus }}+r_{C}^{2} F / g}{\alpha q_{1} H r_{B}^{2} \eta} \quad \text { and } \quad \omega^{*}=\frac{r_{C} F}{\alpha q_{1} H r_{B}^{2} \eta} .
$$

This closed form solution for the dynamics of the bolus provides us with considerable insight into the behavior of the system. Considering Equation (4) in the limit of small and large times leads to two asymptotic dynamical regimes.

When $t / t^{*} \ll 1$, the solution for $\omega$ reduces to an inertial regime of constant acceleration, governed by the total inertia of the system and independent of the liquid viscosity :

$$
\dot{\omega} \sim \frac{\omega^{*}}{t^{*}}=\frac{r_{C} F}{I_{t o t}+I_{\text {bolus }}+r_{C}^{2} F / g} .
$$

When $t / t^{*} \gg 1$, the angular velocity $\omega$ tends to a constant value governed by the viscosity of the liquid, defining a viscous regime :

$$
\omega \sim \omega^{*}=\frac{r_{C} F}{\alpha q_{1} H r_{B}^{2} \eta} .
$$

Furthermore, the oral transit time $t_{\text {end }}$ of the bolus can be deduced from Equation (4). Integrating the expression for $\omega(t)$ with respect to time and setting $\theta\left(t=t_{\text {end }}\right)=\theta_{\text {end }}$ leads to the following equation for $t_{\text {end }}$ :

$$
\frac{t_{\text {end }}}{t^{*}}+e^{-t_{\text {end }} / t^{*}}=1+\frac{\theta_{\text {end }}-\theta_{0}}{\omega^{*} t^{*}} .
$$

This analysis reveals that the oral dynamics of the bolus is a two-stage pro225 cess consisting first of an inertial regime dominated by total system inertia, possibly followed by a viscous regime dominated by liquid viscosity, with $t^{*}$ being the characteristic inertial-viscous transition time between the two regimes. 
Comparing the oral transit time $t_{\text {end }}$ of the bolus with this characteristic transition time leads to a dimensionless quantity $t_{e n d} / t^{*}$ that measures the predominance of the viscous regime over the swallow. In other words, $t_{e n d} / t^{*}$ quantifies the relative importance of viscous effects over inertial effects during the swallow i.e. the sensitivity of the transit time to viscosity. In Section 5, we will investigate how $t_{\text {end }}$ and $t_{\text {end }} / t^{*}$ vary with the driving force and system inertia, from which we will draw physiological conclusions. Furthermore, the inertial and 235 viscous regimes will allow us to explain the behavior of shear-thinning liquids.

\section{Results}

4.1. Calibrating the inertia of the in vitro experiment using in vivo observations

As mentioned in the theory section, it is quite difficult to estimate the inertia of all the organs moving during swallowing, so the approach of lumping these

240

$F=1.4-2 \mathrm{~N}$, corresponds to an applied pressure of about $10 \mathrm{kPa}$, consistent with in vivo measurements from several past studies (Yokoyama et al., 2014, Nicosia et al. 2000, Youmans and Stierwalt, 2006). This in vitro configuration is therefore selected to reproduce the in vivo observations. Other in vitro configinto an effective system inertia $I_{t o t}$ was privileged. To this end, the bolus oral transit time predictions of the full theoretical model were first matched with the in vivo measurements obtained with Newtonian liquids, without resorting to in vitro results. Considering driving forces around $2 \mathrm{~N}$, this procedure led to a moment of inertia $I_{t o t}=1.15 \cdot 10^{-3} \mathrm{~kg} \mathrm{~m}$.

Figure 3 a shows that the in vitro experimental results at $I_{t o t}=1.15$. $10^{-3} \mathrm{~kg} \mathrm{~m}^{2}$ match indeed the in vivo results in terms of bolus exit velocities. In the figure, the circles represent the in vitro results obtained using different driving forces and the squares represent in vivo results. A good agreement is also observed for bolus transit times and their sensitivity to viscosity, as shown by the blue squares in Figure $3 \mathrm{~b}$. The force range considered in this study, namely urations with different moments of inertia have been tested, but they compared 
less favourably with in vivo observations. These results are not included here for brevity, but will be discussed in Section 5

\subsection{Newtonian fluids}

In Figure 4 , the in vivo and the in vitro swallow of low viscosity Newtonian liquids of similar viscosity are qualitatively compared. It can be observed that the movement of the roller follows very well the peristaltic action of the tongue that propels the bolus.

Figure $3 \mathrm{~b}$ presents the effect of liquid viscosity and applied force on the oral transit times. As expected, the transit time increases with the liquid viscosity. Lower driving forces lead to longer transit times and increased sensitivity to viscosity, as demonstrated by the higher slope of the curves. Conversely, we have also observed (in experiments not shown here for brevity) that increasing the system inertia also leads to longer transit times but coupled with lower sensitivity to viscosity. These results will be interpreted in light of the theoretical

270 model in Section 5 .

Figure 5 compares the time evolution of the in vitro roller angular velocity (solid lines) to predictions from the full theoretical model for three Newtonian liquid viscosities (dotted lines), using the parameters selected in the previous section $I_{t o t}=1.15 \cdot 10^{-3} \mathrm{~kg} \mathrm{~m}^{2}, F=2 N$. The agreement between experi275 ments and theory is excellent. The results obtained using TUC solutions will be discussed in the next section.

During in vitro experiments, low viscosity Newtonian liquids were often observed to leak ahead of the bulk of the liquid in an uncontrolled manner, before the swallow was triggered, as depicted in the inset of Figure 3 a. Interestingly, this phenomenon is suggestive of the uncontrolled swallows observed in some dysphagic subjects when drinking Newtonian thin liquids. 


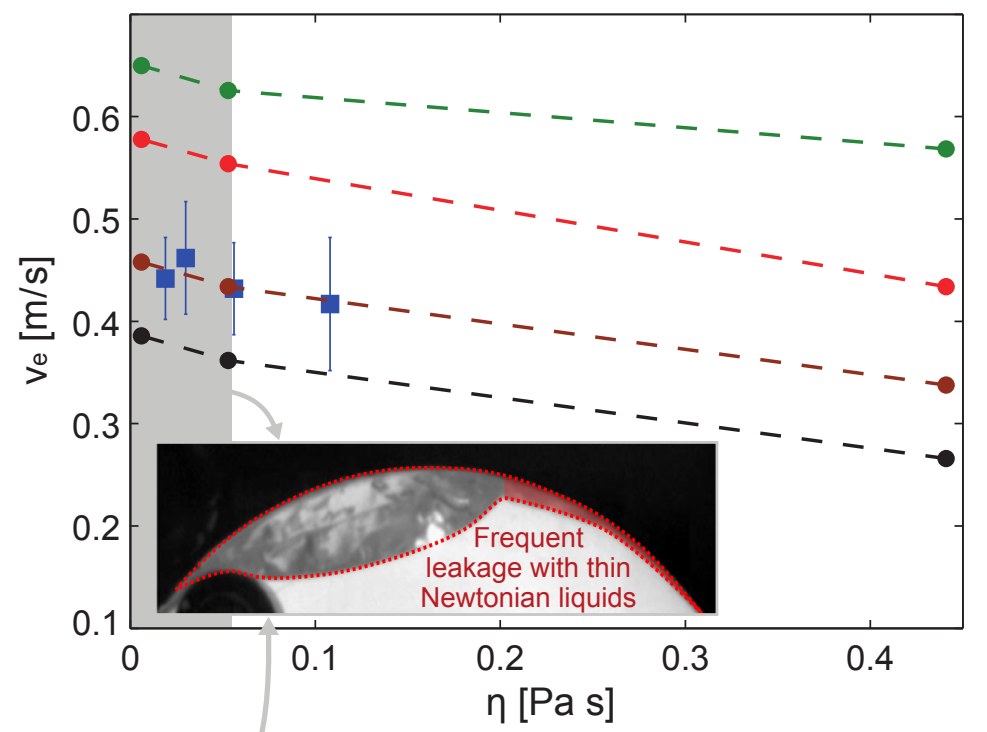

(a)

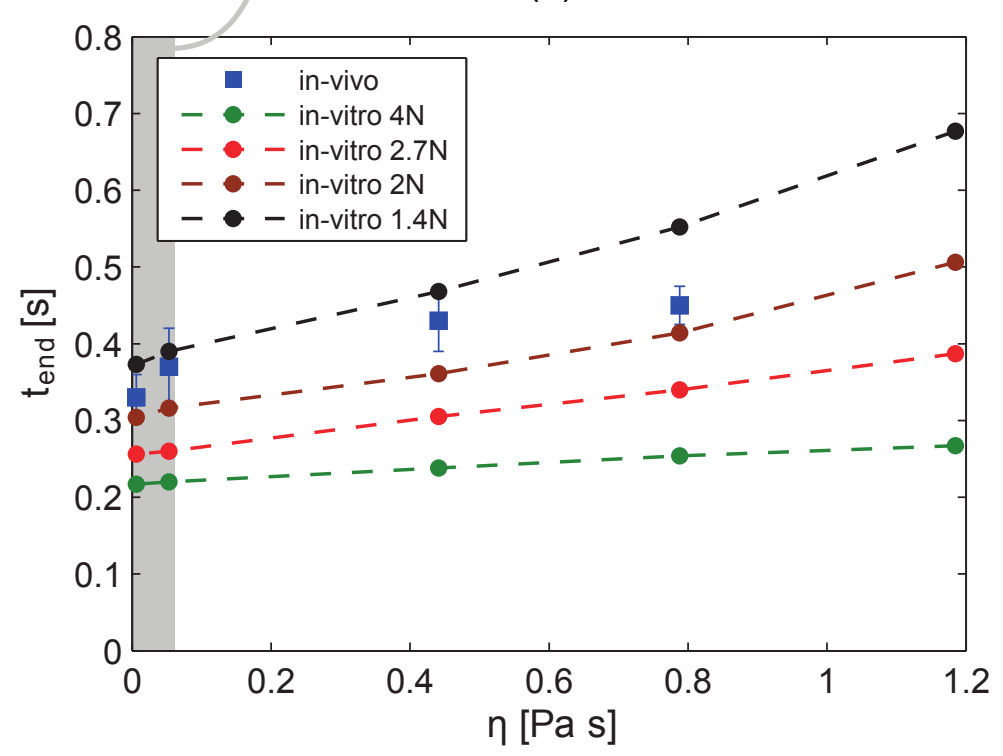

(b)

Figure 3: Comparison between in vivo observations (blue squares) and in vitro experiments (coloured dots) using Newtonian liquids and a moment of inertia $I_{\text {tot }}=1.15 \cdot 10^{-3} \mathrm{~kg} \mathrm{~m}$. Various driving forces are indicated using different colours and dotted lines are used to interpolate the results from the in vitro experiments. (a) Effect of the liquid viscosity on the velocity at the end of the oral phase $v_{e}$. The grey area indicates the range of viscosity producing uncontrolled leakage of the bolus befor 6 the swallow is triggered. A typical snapshot of this phenomenon is shown in the inset of the figure. (b) Effect of the liquid viscosity on the transit time $t_{\text {end }}$. 


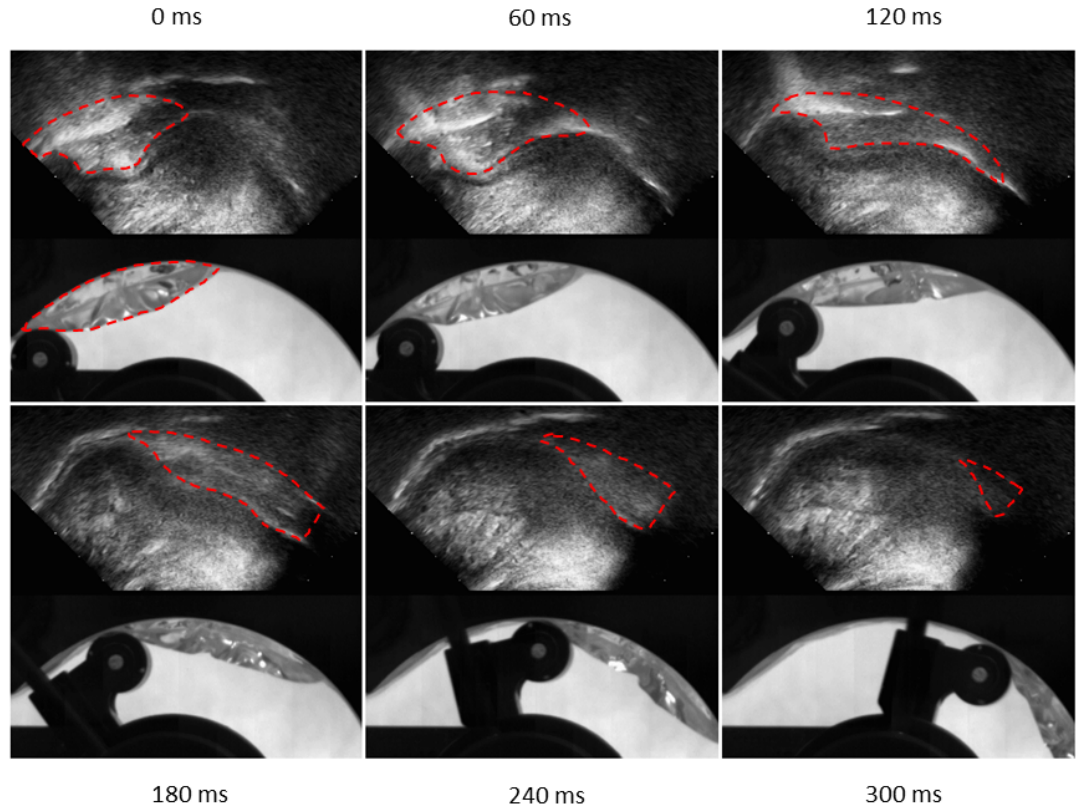

Figure 4: Comparison between the in vivo swallow of an aqueous molasses solution $(\eta=9$ $\mathrm{mPa} s)$ and the artificial swallow of an aqueous glycerol solution $(\eta=6 \mathrm{mPa} \mathrm{s})$ using $I_{\text {tot }}=$ $1.15 \cdot 10^{-3}$ and $F=2 \mathrm{~N}$. 


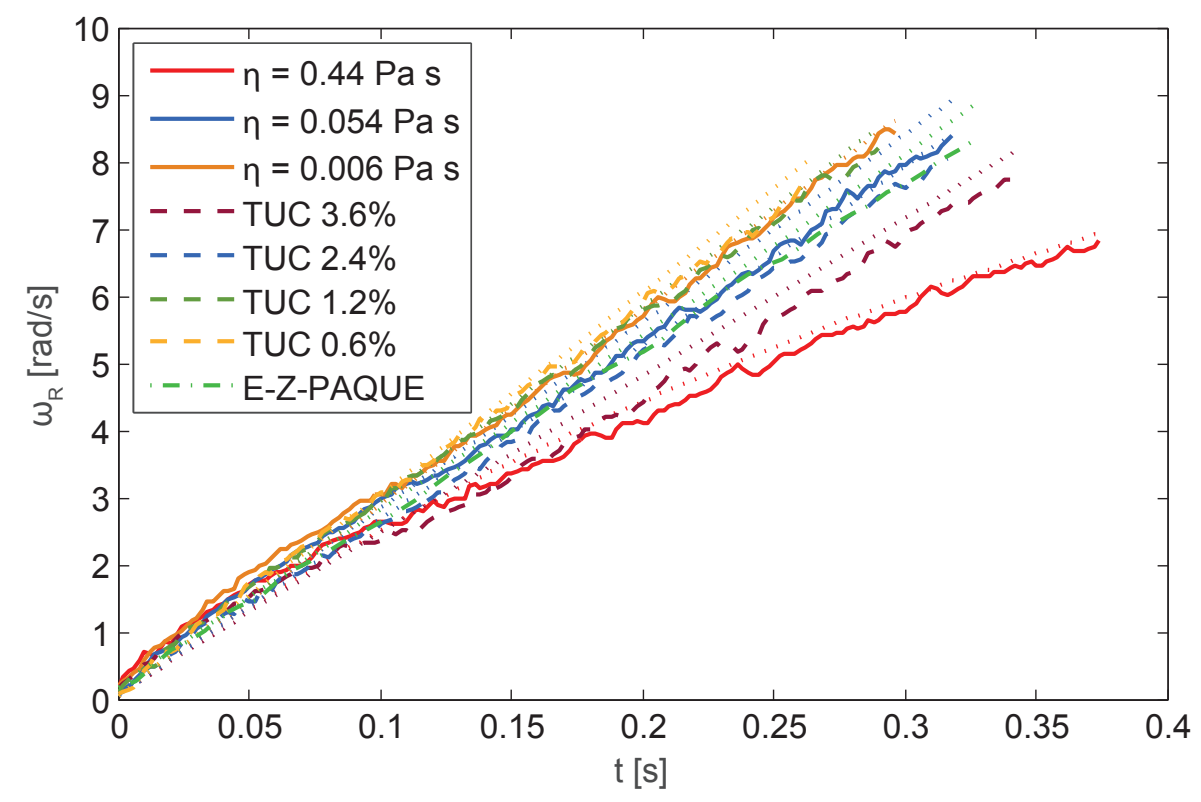

Figure 5: Comparison of the evolution of the angular velocity with time for different Newtonian solutions of low to intermediate viscosity (solid lines), for TUC solutions (dashed lines) and for $41 \% \mathrm{w} / \mathrm{w}$ E-Z-PAQUE (dashed dotted lines). Dotted lines show results from the full theoretical model for comparison. For all curves $I_{t o t}=1.15 \cdot 10^{-3} \mathrm{~kg} \mathrm{~m}{ }^{2}$ and $F=2 \mathrm{~N}$. 


\subsection{Non-Newtonian fluids}

Since most dysphagia thickeners in common use are non-Newtonian, shearthinning materials, it is interesting to test some of these materials in our cal-

during the in vitro swallows were observed for the two thinnest TUC solutions. E-Z-PAQUE and TUC $2.4 \%$ flow more slowly than these solutions, but similarly to one another which is remarkable considering the $42 \%$ higher density of E-ZPAQUE. low has never been observed with shear-thinning liquids, presumably due to their significant zero-shear viscosities. The in vitro results suggest therefore that the main advantage of thickeners, during the oral phase of swallowing, might be to avoid this undesired 'spillover', rather than slowing down the bolus 310 (TUC solutions) was therefore compared to in vivo observations. The effects of varying the solution concentration on the transit time and on the exit velocities are summarized in Figures $6 \mathrm{a}$ and $6 \mathrm{~b}$, respectively. In both figures, the circles represent the in vitro results obtained using different driving forces and the squares represent in vivo results. A driving force $F$ between 1.4 and $2 \mathrm{~N}$ leads to a satisfactory agreement between in vitro and in vivo transit times and oral phase exit velocities for TUC solutions, similar to what was obtained for Newtonian liquids. The system inertia was maintained identical to that for Newtonian fluids.

Following the successful comparison of in vitro and in vivo swallows, the evolution with time of the roller angular velocity for in vitro swallows of TUC solutions of various concentrations was compared to Newtonian results, as shown by the dashed lines in Figure 5. In vitro, the TUC solutions behaved in a surprisingly similar manner to the Newtonian liquids with low or intermediate viscosities, consistent with their high-shear-rate viscosity. Similar dynamics Unlike thin Newtonian liquids, uncontrolled leaking before the in vitro swalflow. 


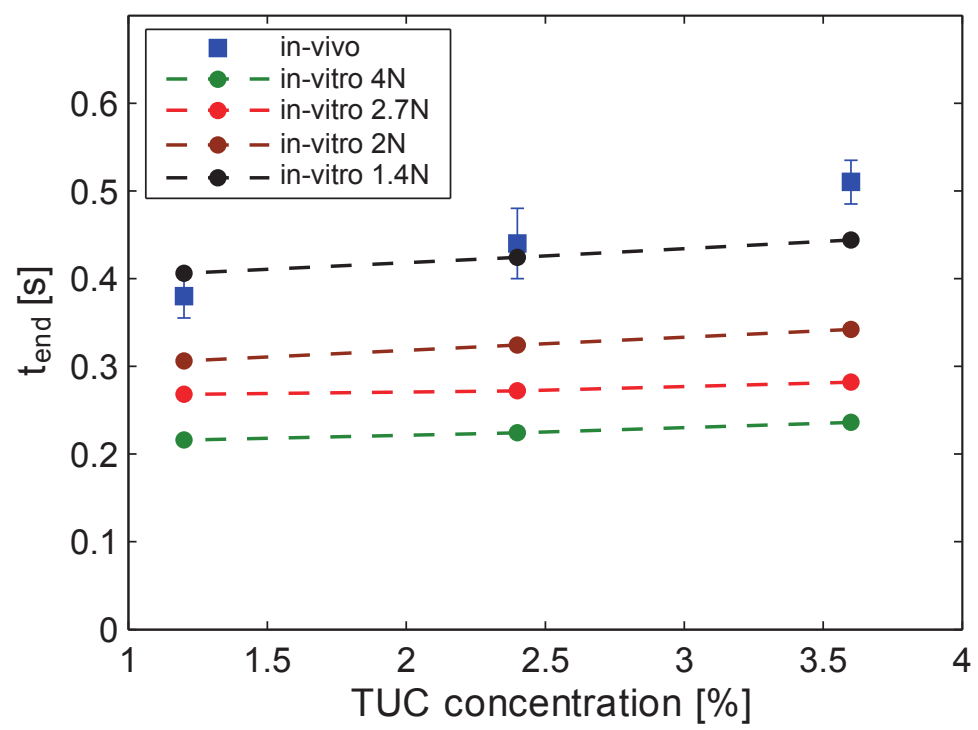

(a)

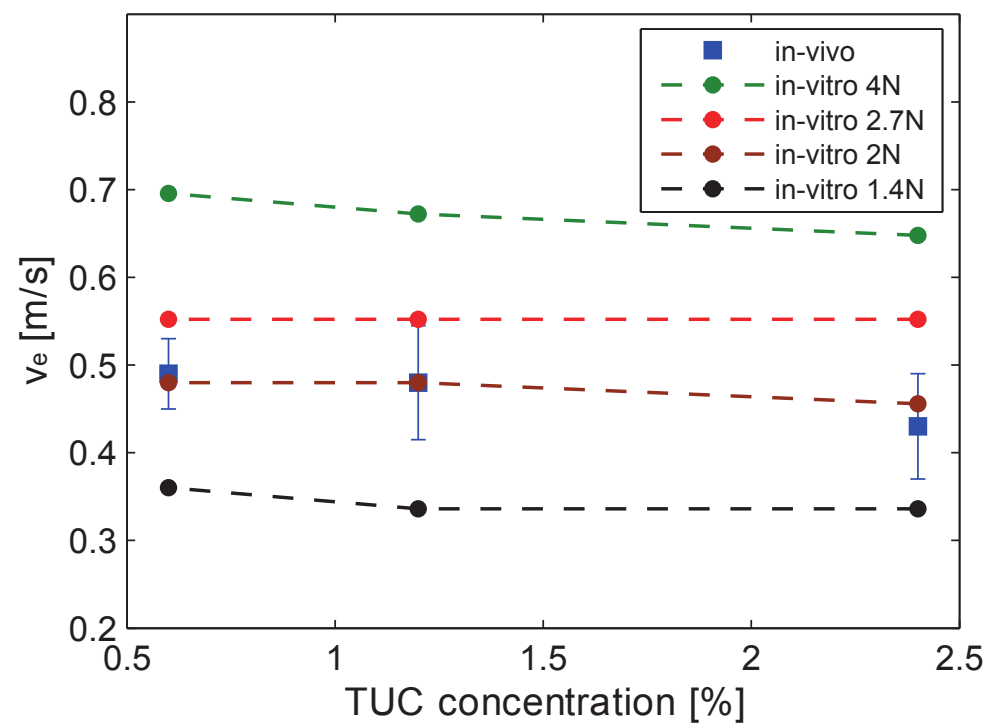

(b)

Figure 6: Comparison between in vivo observations and in vitro results obtained with TUC solutions, various driving forces and $I_{t o t}=1.15 \cdot 10^{-3} \mathrm{~kg} \mathrm{~m}^{2}$. The effect of the TUC concentration on the transit time $t_{e n d}$ and on the velocity at the end of the oral phase $v_{e}$ is represented respectively in panels (a) and (b). 


\section{Discussion}

\subsection{Effects of the applied force and system inertia}

The effects of changing the in vitro system inertia and driving force on transit times and their sensitivity to viscosity were evaluated experimentally in Section 4.2. Some, albeit not large, degree of physiological variation of system inertia and driving force does also occur in humans, for instance between children and adults or as a result of surgery in the treatment of cancer. In this section, the effect of these two parameters is thus investigated in more detail using the theoretical model.

Figure 7 illustrates the predictions of the simplified model developed in Section 3.2 when varying the driving force and the system inertia. Isolines of transit time $t_{\text {end }}$ (panel a) and transit time normalised by the characteristic inertial-viscous transition time $t_{\text {end }} / t^{*}$ (panel b) are represented versus driving force and system inertia, for an intermediate viscosity $\eta=0.44 \mathrm{~Pa}$ s. This figure can be explained as follows: initially the acceleration is relatively constant and inversely proportional to the system inertia. Hence, reducing the ratio of force to inertia increases the transit time (Figure $7 \mathrm{a}$ ). As the system continues to accelerate, the mean shear rate in the fluid increases until the viscous contribution becomes significant when compared with the system inertia, at which point the acceleration fades out and the bolus enters a viscous regime of constant velocity inversely proportional to the liquid viscosity. This time is characterized by $t^{*}$, as seen in Section 3.2, from which the predominance of the viscous regime over the swallow can be measured with $t_{\text {end }} / t^{*}$. Clearly, reducing either system inertia or applied force will cause the transition away from inertially dominated flow to occur at an earlier time, thus increasing the time spent in the viscous regime hence the sensitivity of the transit time to the viscosity (Figure $7 \mathrm{~b}$ ).

The results shown in Figure 7 from the simplified model should only be considered qualitatively. For a quantitative comparison of the full theoretical 


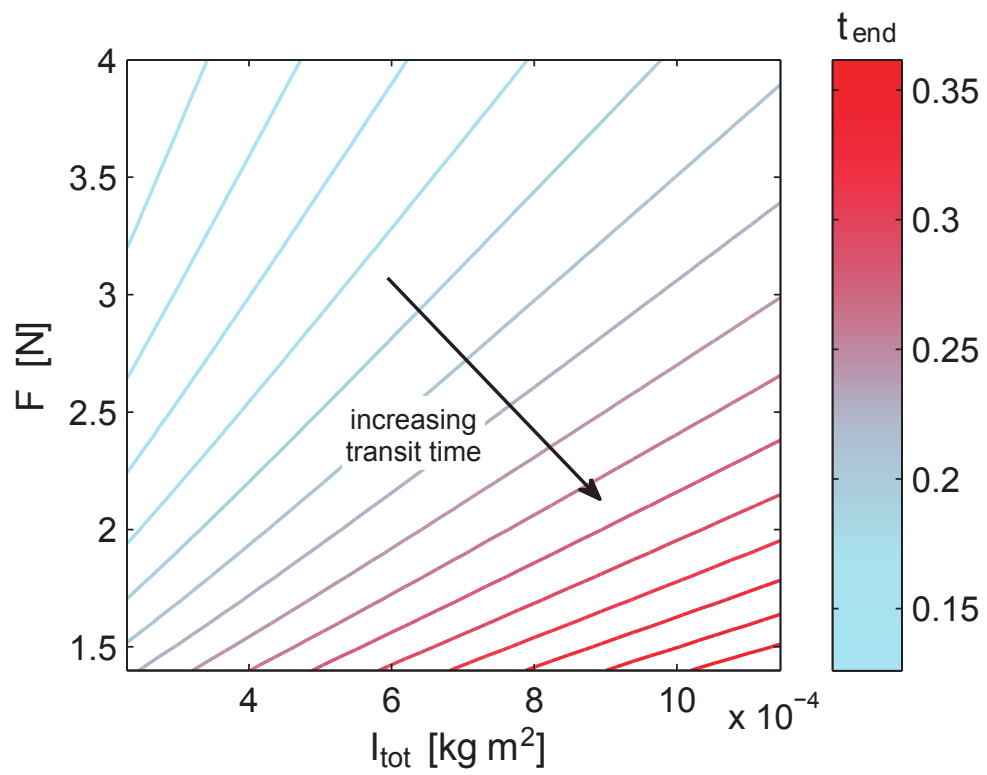

(a)

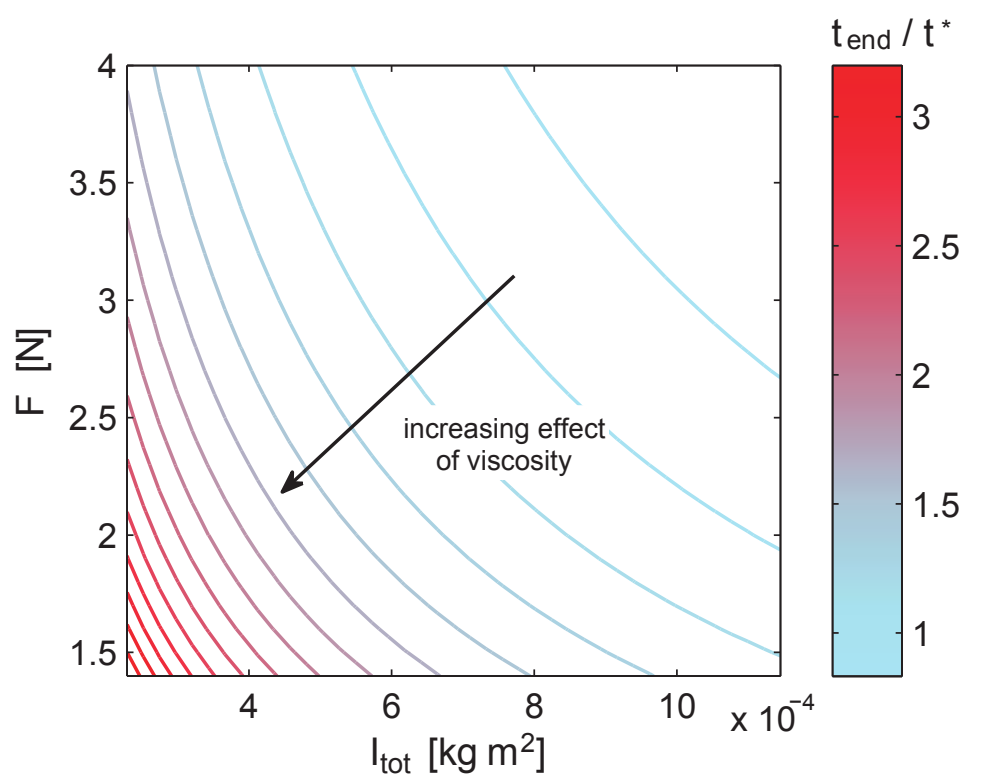

(b)

Figure 7: Contours of (a) $t_{\text {end }}$ and (b) $t_{\text {end }} / t^{*}$ calculated with the simplified model versus system inertia and driving force, for $\eta=0.44 \mathrm{~Pa}$ s. $t_{\text {end }}$ represents the oral transit time of the bolus, while $t_{\text {end }} / t^{*}$ gives a measure of the predominance of the viscous regime over the swallow. The range of values for $I_{t o t}$ and $F$ cover the values used in experiments. 
model from Section 3.1 with the experiments, we show in Figure 8 a comparison of the average bolus transit time and its sensitivity to viscosity predicted by the full theory (hollow circles) with corresponding in vitro results (full circles) for different values of the driving force and system inertia. The average transit time $t_{\text {end }}^{a v g}$ is calculated across the four Newtonian viscosities for which we have both in vivo and in vitro results $(\eta=0.79,0.44,0.053,0.006 \mathrm{~Pa} \mathrm{~s})$, and its sensitivity to 345 viscosity is represented by the transit time ratio $t_{\text {end }}^{\text {high }} / t_{\text {end }}^{\text {low }}$ between the highest and lowest viscosities. This figure not only shows excellent agreement between the full theoretical model and in vitro data, but it also validates the qualitative relevance of the simplified model, since the full theory shows similar trends to Figure 7. Finally, the in vivo results (square dot) confirm that our in vitro experiment with calibrated inertia and physiologically sound driving force leads to realistic transit times and sensitivity to viscosity.

\subsection{Physiological implications}

There are physiologically relevant conclusions to be drawn from the understanding obtained in the previous section, since the applied force can be attributed to tongue strength. Consequently, individuals who have poor tongue strength are likely to experience longer transit times than those with normal tongue strength. In addition, in those individuals the oral transit time of the bolus will be more sensitive to its rheology, meaning that overly thickened boluses are more likely to induce potentially hazardous fluid residue and bad swallowing efficiency, as discussed in the introduction and previously observed in vitro (Hayoun et al. 2015).

Another area that warrants a comment is the widespread belief amongst clinicians that fluid density is an important parameter in determining swallowing dynamics. Our study shows that, although inertia is important for a significant proportion of the unsteady swallowing flow, it is not only the fluid inertia but also the system inertia that matters. Moreover, since $I_{t o t} \sim 10^{-3} \mathrm{~kg} \mathrm{~m}^{2}$ is 


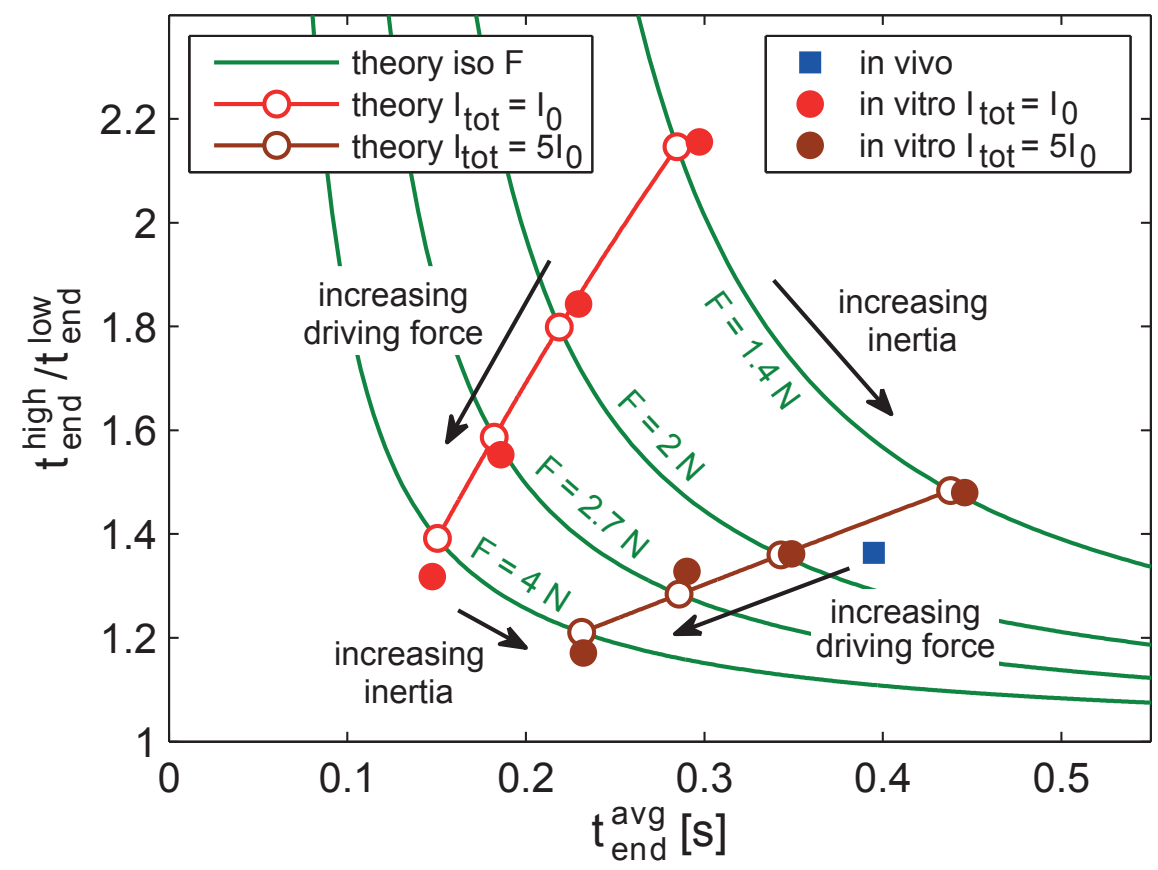

Figure 8: Comparison of the average bolus transit time and its sensitivity to viscosity predicted by the full theory (hollow circles) with corresponding in vitro results (full circles) for different values of the driving force and system inertia. The average transit time $t_{\text {end }}^{\text {avg }}$ is calculated across four Newtonian viscosities $(\eta=0.79,0.44,0.053,0.006 \mathrm{~Pa} \mathrm{~s})$, and its sensitivity to viscosity is represented by the transit time ratio $t_{\text {end }}^{\text {high }} / t_{\text {end }}^{\text {low }}$ between the highest and lowest viscosities. $I_{0}=2.29 \cdot 10^{-4} \mathrm{~kg} \mathrm{~m}{ }^{2}$ represents the base moment of inertia of the device without the offset masses and $5 I_{0}=1.15 \cdot 10^{-3} \mathrm{~kg} \mathrm{~m}^{2}$ is equal to the calibrated value of the inertia. Iso-force and iso-inertia curves from the full theory are also represented, using continuous lines. In vivo results are indicated by a blue square. 
at least an order of magnitude greater than $I_{\text {bolus }} \sim 2 \cdot 10^{-5} \mathrm{~kg} \mathrm{~m}^{2}$, we find no evidence that changing the bolus density would have a significant effect on swallowing, at least from the perspective of bolus propulsion in the mouth.

370 This is confirmed by our experimental results with E-Z-PAQUE, which rheology is in between TUC solutions at 1.2 and $2.4 \%$, but has $42 \%$ higher density. Despite this substantial increase in bolus density, it shows in Figure 5 very similar dynamics to the TUC solutions. These observations support the use of thickeners that match the rheological properties of contrast agents used to 375 diagnose dysphagia. There could be other effects that we do not consider in the current study where fluid inertia is indeed important. One such area could be the inertial flow of the bolus over the epiglottis (Burbidge et al. 2016).

\subsection{Behavior of shear-thinning thickeners for the treatment of dysphagia}

In vitro results from Figure 5 have shown that TUC solutions display transit times similar to low-mid viscosity Newtonian liquids. This behavior is now explained in light of the inertial and viscous regimes identified by the model. At the beginning of the in vitro swallow, system inertia dominates over viscous dissipation, hence the high viscosity of shear-thinning thickeners at low shear rates does not influence significantly the transit time. It is only during the 385 subsequent viscous regime that the viscous contribution becomes predominant. As a result, the transit time of shear-thinning thickeners is controlled by their high-shear-rate viscosity. An estimate for the wall shear rates $\dot{\gamma}$ experienced in the bolus during the viscous regime can be retrieved from the oral phase exit velocities of TUC solutions (Figure 6b). Considering the laminar velocity profile for a circular pipe of a shear-thinning fluid with rheology $\eta=K \dot{\gamma}^{n-1}$ and the power-law coefficients summarized in Table 1, a wall shear rate $\dot{\gamma} \sim 500-800 \mathrm{~s}^{-1}$ can be calculated for different TUC concentrations. Figure 2 reveals that at these shear rates TUC solutions have an apparent viscosity equivalent to that of low to mid viscosity Newtonian liquids, explaining why they flow similarly. 
Table 1: Power law model parameters for the shear-thinning liquids at $22^{\circ} \mathrm{C}$.

\begin{tabular}{ccccc}
\hline Fluid & $\% \mathrm{w} / \mathrm{w}$ & $\rho\left(\mathrm{kg} / \mathrm{m}^{3}\right)$ & $K\left(\right.$ Pas $\left.^{n}\right)$ & $n$ \\
\hline E-Z-PAQUE & $41 \%$ & 1420 & 1.774 & 0.392 \\
TUC & $0.6 \%$ & 1000 & 0.328 & 0.463 \\
TUC & $1.2 \%$ & 1000 & 2.030 & 0.274 \\
TUC & $2.4 \%$ & 1000 & 9.502 & 0.152 \\
TUC & $3.6 \%$ & 1000 & 12.35 & 0.188 \\
\hline
\end{tabular}
inertia, possibly followed a viscous regime in which the viscosity governs the constant velocity of the bolus. Hence, the flow of shear-thinning thickeners is 
governed by their high-shear-rate viscosity, and they indeed behave similarly to low-viscosity Newtonian liquids. However, these liquids are not affected by an unwanted spillover effect observed with thin Newtonian liquids, and as such they provide better control of the bolus before the swallow is triggered.

Another conclusion from our study is that lower driving forces result in longer transit times and higher sensitivity to viscosity. From a physiological standpoint, this means that individuals with poor tongue strength are more likely to experience longer transit times and might be more sensitive to overly thickened boluses, resulting in bad swallowing efficiency.

Finally, the study reveals that the effect of system inertia is significant, hence in future research the anatomical differences between adults and children should be quantified and inertia differences considered when designing dysphagia thickeners. On the other hand, the density of the bolus is shown to play a negligible role in its dynamics. This suggests that the effect of the higher density of contrast agents can be neglected, and contrast agent that are rheologically matched to thickener solutions present the same oral swallowing dynamics.

Insights provided by in vitro experiments and theory can guide the design of novel thickener formulations and help identifying the most promising formulations for clinical evaluations.

\section{Acknowledgements}

This study was funded by Nestlé Research. The authors would like to thank the NRC workshop for designing and building the model swallowing experiment used in this study and Mrs. B. Aubert for the technical assistance with the ultrasound measurements.

\section{Conflict of interest statement}

The authors have no conflicts of interest to declare. 
This appendix describes the way the dissipation force $F_{d}(\rho, \eta, \omega)$ was computed from a computational fluid dynamics (CFD) simulation of the flow within the bolus. In the case of a very flexible membrane, such as the one considered in this study, the drag force has been shown in Takagi and Balmforth (2011) to be provided purely by the fluid pressure and determined by the flow within the bolus. While Hayoun et al. (2015) have considered a linear dependency of $F_{d}$ in $\omega$, other studies (Brown and Hung, 1977, Takabatake and Ayukawa, 1982) have shown that this is valid only at low Reynolds numbers $R e$. At higher Re (typically reached for lower viscosity liquids), it becomes necessary to account for inertial effects in the fluid. To that effect, $F_{d}$ was computed using a COMSOL finite element simulation of the bolus flow, in the configuration shown in Figure 9. The Navier-Stokes equations were solved in the reference frame translating at the velocity $r_{B} \omega$ of the bolus, leading to a time invariant geometry. A tangential velocity with horizontal component equal to $-r_{B} \omega$ was imposed at the top wall, while a symmetry condition was imposed at the bottom wall. Equal pressures were imposed at the inlet and outlet. Lengths were normalized by the bolus height $H$, the pressure by $\rho r_{B}^{2} \omega^{2}$, leading to $R e=\rho H r_{B} \omega / \eta$. Integrating the horizontal component of the pressure applied by the fluid to the wall along the contact line with the roller, the non-dimensional dissipation force acting on the roller $f(R e)$ was computed as a single function of Re. This resulted in the following expression for the dimensional force:

$$
F_{d}(\rho, \eta, \omega)=\alpha \rho r_{B}^{2} \omega^{2} H^{2} f(R e)=\alpha \eta^{2} / \rho R e^{2} f(R e)
$$

where $\alpha=10.6$ is a correction factor for the dissipation term that was obtained by fitting the theory to the experimental data. Figure 9 shows that the dimensionless dissipation force depends linearly on the velocity at low $R e$ and quadratically at high $R e$, as expected from a simple scaling analysis. 


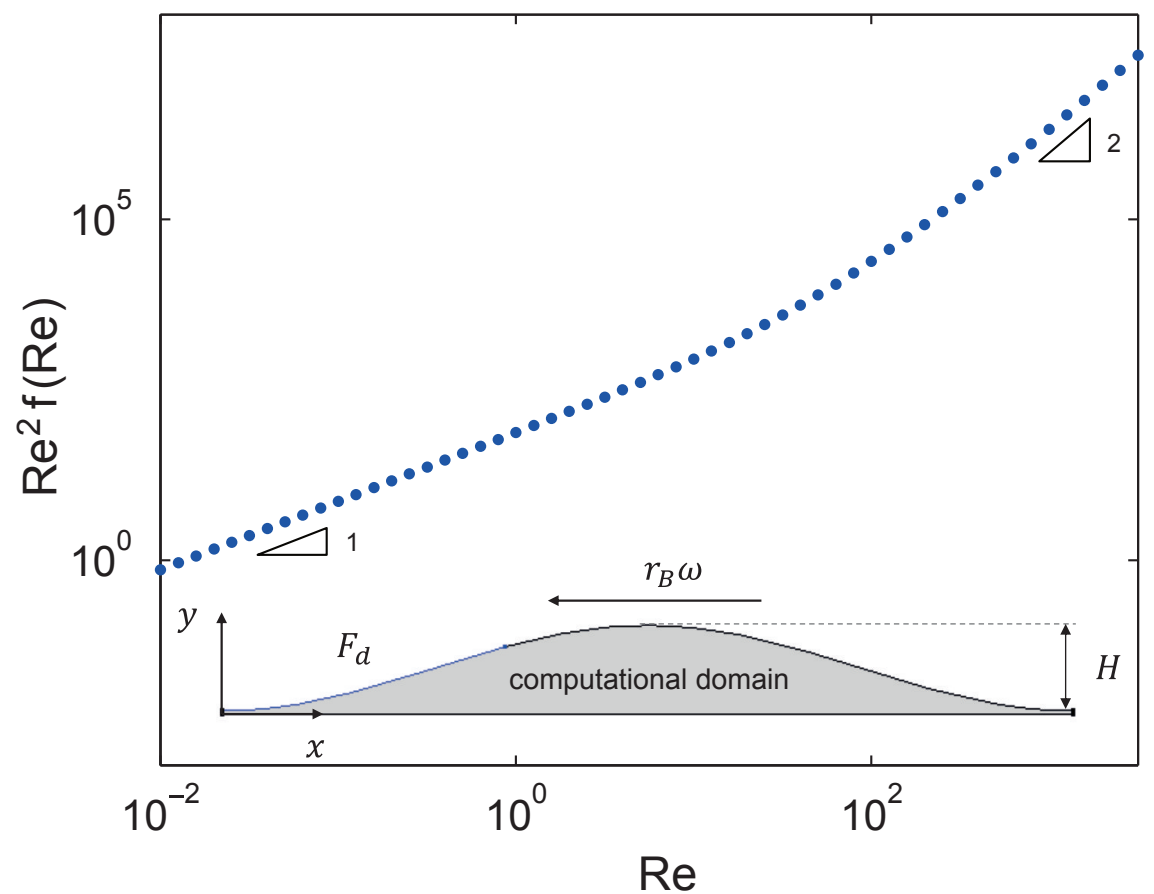

Figure 9: Dimensionless dissipation force $R e^{2} f(R e)$ obtained from the numerical simulation of the bolus flow in the computational domain shown in the inset at different Re. The geometry is time invariant in the reference frame translating at the velocity $r_{B} \omega$ of the bolus. 
Brown, T.D., Hung, T.K., 1977. Computational and experimental investigations of two-dimensional nonlinear peristaltic flows. Journal of Fluid Mechanics 83, $249-272$.

Burbidge, A., Cichero, J., Engmann, J., Steele, C., 2016. A day in the life of the

De Loubens, C., Magnin, A., Verin, E., Doyennette, M., Tréléa, I.C., Souchon, I., 2010. A lubrication analysis of pharyngeal peristalsis: Application to flavour release. Journal of Theoretical Biology 267, $300-311$.

Hayoun, P., Engmann, J., Mowlavi, S., Le Reverend, B., Burbidge, A., Ramaioli, M., 2015. A model experiment to understand the oral phase of swallowing of newtonian liquids. Journal of Biomechanics 48, 3922-8.

Hiss, S.G., Postma, G.N., 2003. Fiberoptic endoscopic evaluation of swallowing. The Laryngoscope 113, 1386-1393.

Koennecke, H.C., Belz, W., Berfelde, D., Endres, M., Fitzek, S., Hamilton, F., Kreitsch, P., Mackert, B.M., Nabavi, D., Nolte, C., Phls, W., Schmehl, I., Schmitz, B., von Brevern, M., Walter, G., Heuschmann, P., the Berlin Stroke Register Investigators, F., 2011. Factors influencing in-hospital mortality and morbidity in patients treated on a stroke unit. Neurology 77, 965-972.

Logemann, J.A., 1998. The evaluation and treatment of swallowing disorders. 470 Current Opinion in Otolaryngology \& Head and Neck Surgery 6, 395-400. 
Mackley, M., Tock, C., Anthony, R., Butler, S., Chapman, G., Vadillo, D., 2013.

The rheology and processing behavior of starch and gum-based dysphagia thickeners. Journal of Rheology 57, 1533-1553.

Newman, R., Vilardell, N., Clavé, P., Speyer, R., 2016. Effect of bolus viscosity on the safety and efficacy of swallowing and the kinematics of the swallow response in patients with oropharyngeal dysphagia: White paper by the european society for swallowing disorders (essd). Dysphagia 31, 232-249.

Nicosia, M.A., Hind, J.A., Roecker, E.B., Carnes, M., Doyle, J., Dengel, G.A., Robbins, J., 2000. Age effects on the temporal evolution of isometric and swallowing pressure. The Journals of Gerontology Series A: Biological Sciences and Medical Sciences 55, M634-M640.

Nicosia, M.A., Robbins, J., 2001. The fluid mechanics of bolus ejection from the oral cavity. Journal of Biomechanics 34, 1537-1544.

Popa Nita, S., Murith, M., Chisholm, H., Engmann, J., 2013. Matching the rheological properties of videofluoroscopic contrast agents and thickened liquid prescriptions. Dysphagia 28, 245-252.

Scheitz, J.F., Endres, M., Heuschmann, P.U., Audebert, H.J., Nolte, C.H., 2015. Reduced risk of poststroke pneumonia in thrombolyzed stroke patients with continued statin treatment. International Journal of Stroke 10, 61-66.

490 Takabatake, S., Ayukawa, K., 1982. Numerical study of two-dimensional peristaltic flows. Journal of Fluid Mechanics 122, 439-465.

Takagi, D., Balmforth, N., 2011. Peristaltic pumping of viscous fluid in an elastic tube. Journal of Fluid Mechanics 672, 196-218.

Weickenmeier, J., Jabareen, M., Le Reverend, B., Ramaioli, M., Mazza, E., 2016. Experimental and numerical characterization of the mechanical masseter muscle response during mastication. subm. . 
Yokoyama, S., Hori, K., Tamine, K.I., Fujiwara, S., Inoue, M., Maeda, Y., Funami, T., Ishihara, S., Ono, T., 2014. Tongue pressure modulation for initial gel consistency in a different oral strategy. PLoS ONE 9.

${ }_{500}$ Youmans, S.R., Stierwalt, J.A., 2006. Measures of tongue function related to normal swallowing. Dysphagia 21, 102-111. 